\title{
Detection of Impurities in Anti-infective Generic Drugs in Brazil by Liquid Chromatography-Mass Spectrometry
}

\author{
Jeancarlo Pereira dos Anjos*1, PhD; Samuel Sherratt ${ }^{2}$ Bsc, PhD; Josiane Dantas ${ }^{1}$ PhD; Valdir Gomes Barbosa \\ Junior'; R. Preston Manson ${ }^{2,3}$; Roberto Badaro', MD, PhD \\ ${ }^{1}$ Health Institute of Technology - SENAI CIMATEC, National Service of Industrial Learning - SENAI; Salvador, Bahia, \\ Brazil; ${ }^{2}$ Elucida Research, Beverly, Massachusetts, USA; ${ }^{3}$ Brigham \& Women's Hospital, Harvard Medical School, Boston, \\ Massachusetts, USA
}

\begin{abstract}
Impurities found in generic medicinesmay contribute to loss of efficacy and adverse side effects when administered to patients suffering from various diseases. Methods of analysis of the quality of drug products are well advanced. Herein, we used Liquid Chromatography with Diode Array Detector coupled to Mass Spectrometry to detect the presence of organic impurities and determine the quantity of the Active Pharmaceutical Ingredient (API) present in representative Antibiotics (2) and Antifungals (2). Possible impurities were detected in some of the generic drugs in both classes of anti-infective drugs. No impurities were detected in the amoxicillin. The compounds 3'-N,N-Di(demethyl) azithromycin (azithromycin impurity $\mathbf{E}$ ) and 3'-De(dimethylamino)-3'-keto azithromycin (azithromycin impurity $\mathbf{N}$ ) were detected in generic azithromycin. For itraconazole, the compounds cis-[2-(2,4-dichlorophenyl)-2-(1H-1,2,4-triazol4-yl-methyl)-1,3-dioxolan-4-yl]methylmethanesulfonate and trans-[2-(2,4-dichlorophenyl)-2-(1H-1,2,4triazol-1-yl-methyl)-1,3-dioxolan-4-yl]methylmethanesulfonate, besides a third substance identified as 2(2-Butyl) -4-\{4-[4-(4-methyloxy-phenyl)-piperazin-1-yl]-phenyl\}-2,4-dihydro-[1,2,4]-triazol-3-one were detected as possible impurities. Interestingly, an additional peak was noted in the chromatogram for the generic fluconazole, in addition to the peak of the API; however, none of known impurities of fluconazole were identified. We conclude that tests in addition to bioequivalence measurements may be required to assess post-market generic quality. Such surveillance of generic quality should be performed routinely. Keywords: Generic Drugs. Mass Spectrometry. Drug Impurities.
\end{abstract}

Impurities may be present in medicinal products generated from poorly controlled manufacturing conditions,poor-quality substrates and/or API synthesized by a process different from than the certified synthesis pathway $[1,2]$. The impact of the use of low-quality drugs is related to the adverse effects caused by the presence of undesirable substances, such as impurities and worse, it may contain theincorrect Active Pharmaceutical Ingredient (API) [3].

The adulteration of drugs has been grouped into five categories: (i) copies of the authentic medicine, butwith changes in the quantityof API; (ii) products with incorrect API, which may be of Received on 20 March 2019; revised 22 May 2019.

Address for correspondence: Dr. Jeancarlo Pereira dos Anjos. Avenida Orlando Gomes, N. 1845, Piatã. Zip Code: 41650010. Salvador, Bahia, Brazil. Phone: (+55 71) 3879-5501.Email: jeancarlo.anjos@fieb.org.br. This work was funded from SENAI-CIMATEC Research Center, Bahia, Brazil and the Minister of Health of Brazil.

J Bioeng. Biotech. Appl. Health 2019;2(2):36-46.

(C) 2019 by SENAI CIMATEC. All rights reserved. inferiorquality; (iii) preparations with an absence of API; (iv) medicines with very high or very low API content; (v) contamination with known and/ or unknown impurities [4,5].

Due to lower costs, the use of generic drugs has been promotedin many countries [6]. Policies to reduce costs and increase the availability of medicineshave contributed to the preventionofdrug shortages, in addition to improving accessibility to drugs invarious therapeutic classes, especially in developing countries [7-9]. Analytical methods for rapid and accurate drug testingare necessary to combat the increasing number of adulterated drugs as well assophisticated counterfeits [10-15]. The deployment of such analytical technology will have an important role indetecting drug tampering. The safety of medicines depends on the absenceof impurities and accurate API levels. Effective and reproduciblemethods fordetecting drug impurities and API levels may provide insightsinto the quality of generic drugs available in the global marketplace [16,17]. 
High-performance liquid chromatography (HPLC) has been a common technique for the determination of impurities in pharmaceutical products [11]. For basic identification and quantification of the components of the sample, $\mathrm{UV} / \mathrm{Vis}$ spectrophotometry data is gathered using a diode array detector (DAD). Additionally, this analytical technique can be coupled to mass spectrometry (MS) for the structural identification of components of interest such as impurities resulting from improper synthesis $[18,19]$. Thus, chromatographic techniques have been powerful tools in the analysis and characterization of adulterated medicines because they assess the composition of the analyzed sample [10].

Herein we analyzed the generic antibiotics amoxicillin trihydrate, azithromycin dihydrate, and the generics antifungals fluconazole and itraconazole by high-performance liquid chromatography to detect the presence of impurities in the products.

Generic drugs were provided by the Minister of Health of Brazil (MH). The generic medicines provided were selected from batches stored at the $\mathrm{MH}$ that were ready to be distributed to the population. Each generic and branded drug tablet or capsule was prepared for HPLC-DAD-MS analysis by extracting API in methanol, with the exception of amoxicillin trihydrate (in water). The analyses were tested in collaboration with other laboratories at a referral laboratory in Boston, MA - USA (personal communication from Elucida Research). Certified standards of the API for the antibiotics (amoxicillin trihydrate and azithromycin dihydrate), were purchased from Toronto Research Chemicals (Toronto, Canada) for comparative analysis.

API Impurity and Quantification Analysis were performed as follows: Stock solutions were prepared at 1-5 mg mL-1 in an aqueous or organic solvent to match the corresponding sample preparation for each compound. Analytical curves for each compound were constructed at the following concentrations: $0.05,0.1,0.2,0.25$ and $0.5 \mathrm{mg} \mathrm{mL}-1$.

Tablets were crushed using an Agate mortar and pestle and solubilized in an aqueous or organic solvent at $1-5 \mathrm{mg} \mathrm{mL}-1$, depending on the nature of the API. For products delivered in gelatin capsule form, the two halves of the capsule were separated and the contents were solubilized in aqueous or organic solvent. Solutions were stirred at least 4 hours to ensure uniform distribution of material. The active pharmaceutical ingredient (API) was then separated from insoluble excipient by centrifugation at room temperature for 30 minutes. The supernatant (containing the API) was then removed and stored at $4^{\circ} \mathrm{C}$ before HPLCDAD-MS analysis.

Each sample was diluted down to a nominal concentration of 0.2 or $0.5 \mathrm{mg} \mathrm{mL}-1$, and then the actual concentration was determined based on the analytical curve. Results were reported as a percentage of expected concentration compared to standard solutions as well as compared to branded formulations when applicable. For impurity analysis, samples were run at $0.5 \mathrm{mg} \mathrm{mL}-1$.

Samples and standard solutions were run on an Agilent 1260 Series LC, equipped with a diode array detector (DAD) and an Agilent 6120B MSD for mass spectrometry analysis. All samples were run on a Poroshell 120 EC-C18 4.6 X $100 \mathrm{~mm}$ $2.7 \mu \mathrm{M}$ column. For the evaluation of impurities, the drug samples were analyzed using the mass spectrometric detector, described above, in SCAN mode $(\mathrm{m} / \mathrm{z} 100$ to 800$)$. For the quantification of the APIs, the diode array detector (DAD) was operated at the wave lengths according to the optimal absorbance of electromagnetic radiation for each analyte.

Figure 1A shows the chromatograms and mass spectrum for the pure azithromycin dihydrate standard. In Figure 1A, the peak at $4.797 \mathrm{~min}$ corresponds to azithromycin, as evidenced by the characteristic mass spectrum and associated molecular weights (Figure 1B). The molecular weight of azithromycin is $749 \mathrm{~g}$ mol- 1 .

Figure 2A shows the LC-MS analysis, where the peak at $4.832 \mathrm{~min}$ corresponds to generic azithromycin while the 6.052 min peak corresponds to a potential impurity not seen in the standard formulation. In Figure 2B, we show the mass spectrum analysis of this peak, which 
Figure 1. Chromatogram obtained by LC-MS (SCAN mode) for pure azithromycin dihydrate (A) and mass spectrum of pure azithromycin dihydrate (B).
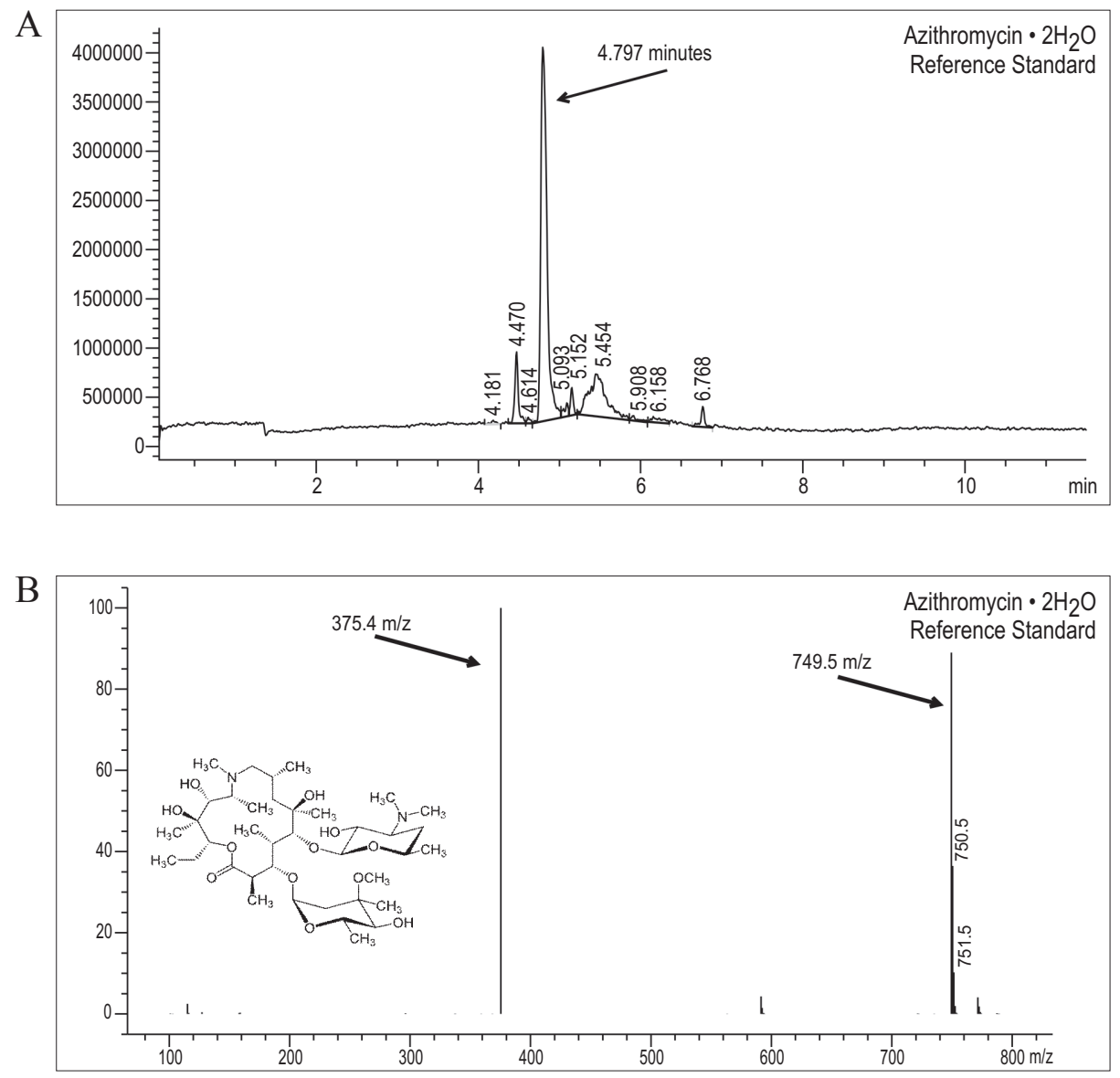

revealed the characteristic of the mass-to-charge ratio associated with azithromycin, which also matches the pattern of the standard azithromycin. The molecular weight of azithromycin is $749 \mathrm{~g}$ mol-1, as shown in Figure 2B. Also, in Figure 2A, we noted several smaller peaks, which were also seen in the standard formulation.

The peak seen at 6.052 min (Figure 2A) did not have a corresponding peak in the standard formulation. Analysis of the mass spectrum of this peak (Figure 3) revealed a chemical entity with a molecular weight around to $720 \mathrm{~g}$ mol-1. According to Chang et al. (2015) (20), there are two possible impurities in the azithromycin with similar molecular weight. One of the molecules, azithromycin EP impurity E (3'-N,N-Di(demethyl) azithromycin), has a molecular weight of $720.9 \mathrm{~g}$ mol-1 (Figure 4A).Anothermolecule, azithromycin EP impurity N (3'-De(dimethylamino)-3'-keto azithromycin), has a molecule weight of $719.9 \mathrm{~g}$ mol-1 (Figure 4B).

The reference and generic formulations of amoxicillin hydrated were analyzed for impurities using LC-MS as previously described. The amoxicillin trihydrate standard (2.992 $\mathrm{min})$ and both the reference $(2.985 \mathrm{~min})$ and generic $(2.982$ min) amoxicillin trihydrate show a similar peak as shown in Figure 5. The mass spectrum of the standard, the reference and the generic confirmed that the only peak observed in both chromatograms correspond to amoxicillin hydrated. Nevertheless, no other peaks were noted. Thus, it confirms that no impurities were encountered in both reference and generic amoxicillin. 
Figure 2. Chromatogram obtained by LC-MS (SCAN mode) for generic azithromycin dihydrate (A) and the mass spectrum of 4.832 min peak for generic azithromycin dihydrate (B)
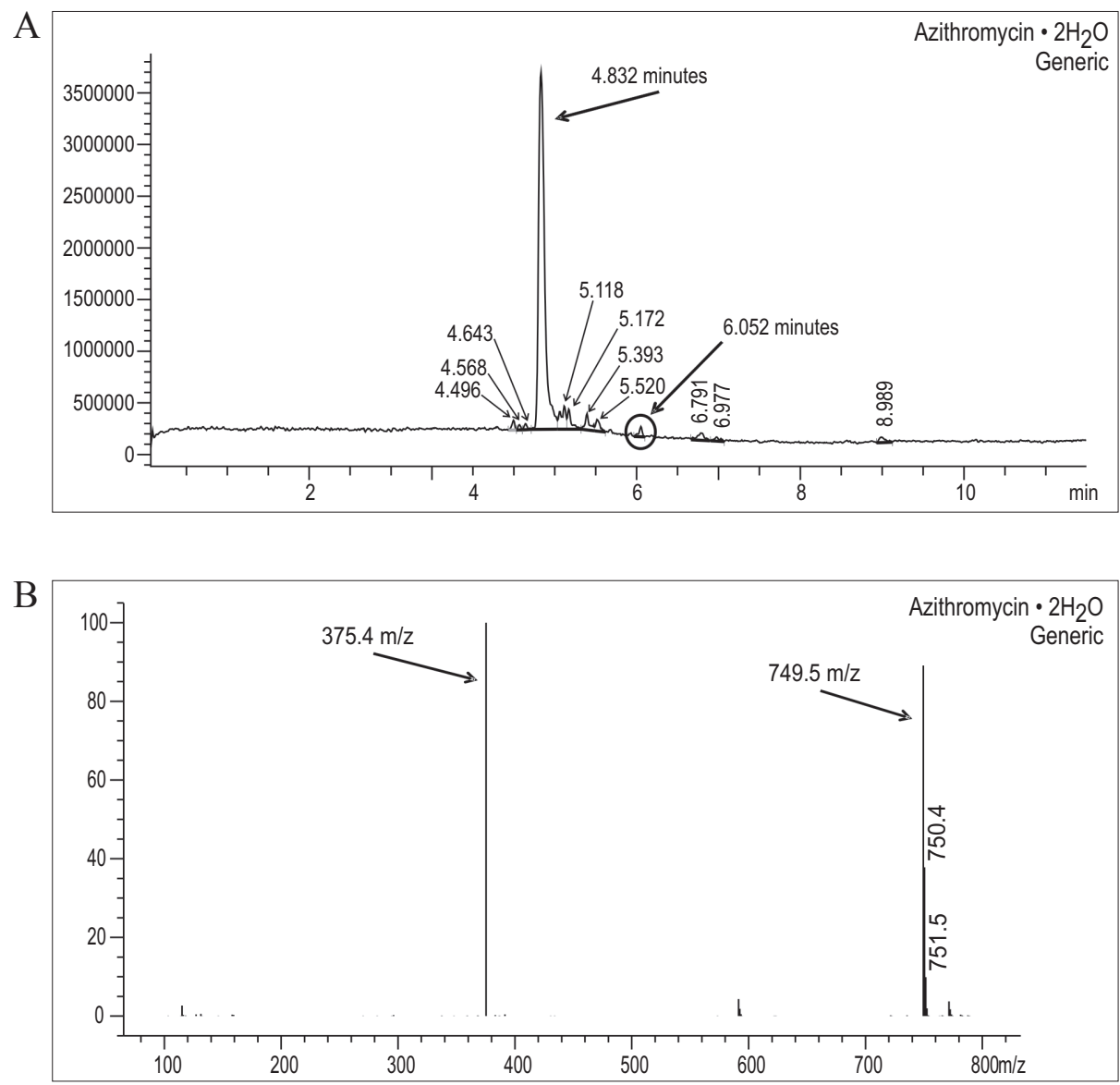

Figure 3. Mass spectrum of 6.052 min peak for generic azithromycin dihydrate. The pattern of this mass spectrum indicates a chemical entity with a molecular weight around $720 \mathrm{~g}$ mol-1 that was not seen in the standard formulation.

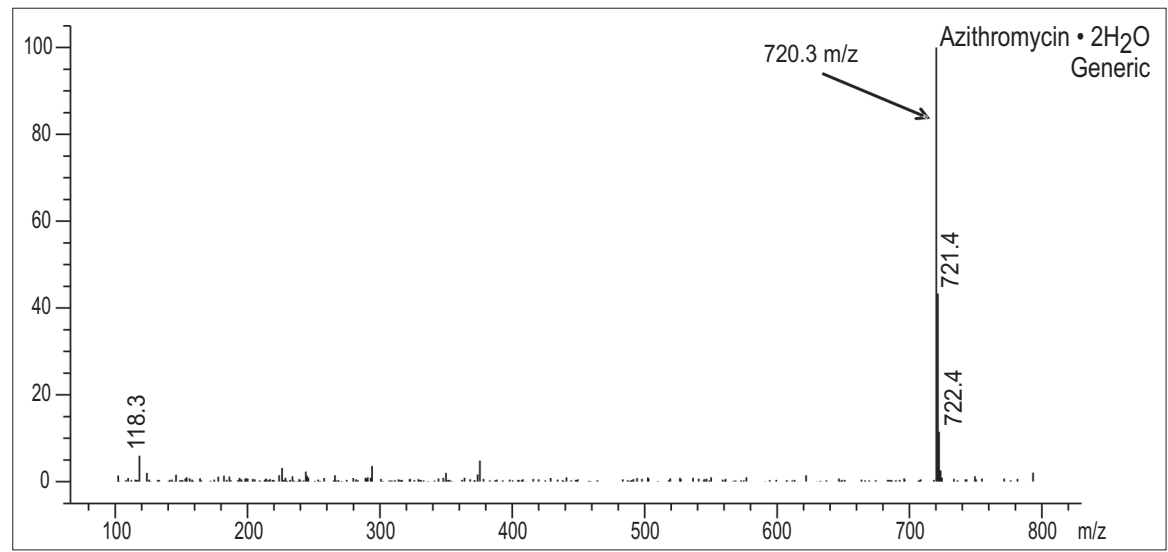


Figure 4. Chemical structure of possible impurities found in the generic formulation of azithromycin dihydrate. (A) 3'-N,N-Di(desmethyl) azithromycin (CAS\#: 612069-27-9, azithromycin EP impurity E), which has molecular weight of $420.9 \mathrm{~g}$ mol-1 and (B) 3'-Des(dimethylamino)-3'-keto azithromycin (CAS\#: 612069-25-7, azithromycin EP impurity N), which has molecular weight of $419.9 \mathrm{~g}$ mol-1.

A

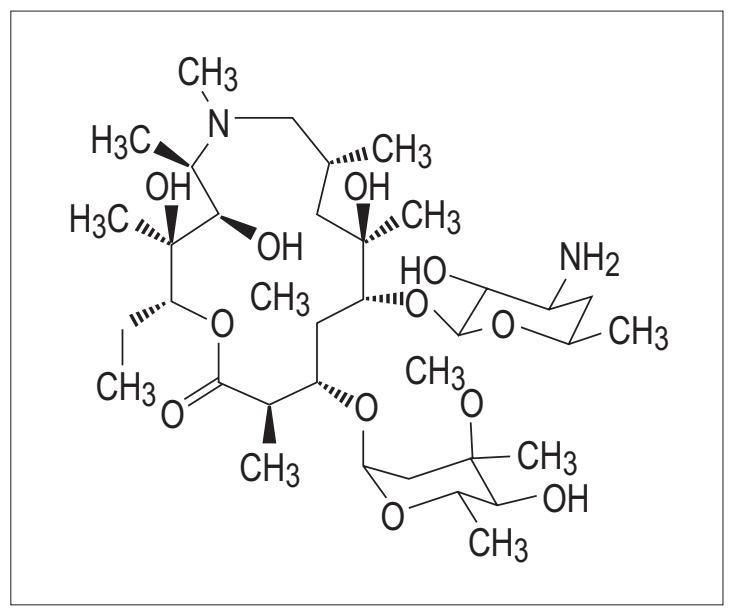

$\mathrm{B}$

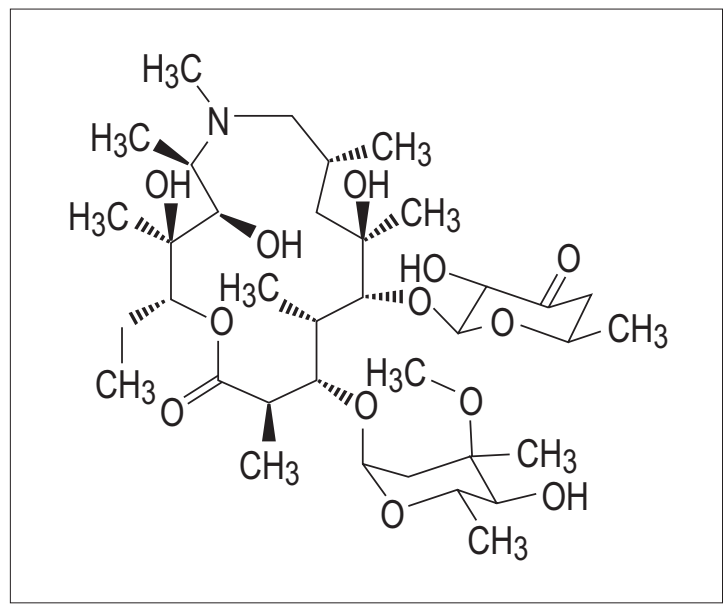

Figure 5. Chromatograms and mass spectrums obtained for (A) standard amoxicillin trihydrate, (B) reference amoxicillin trihydrate and (C) generic amoxicillin trihydrate.

A

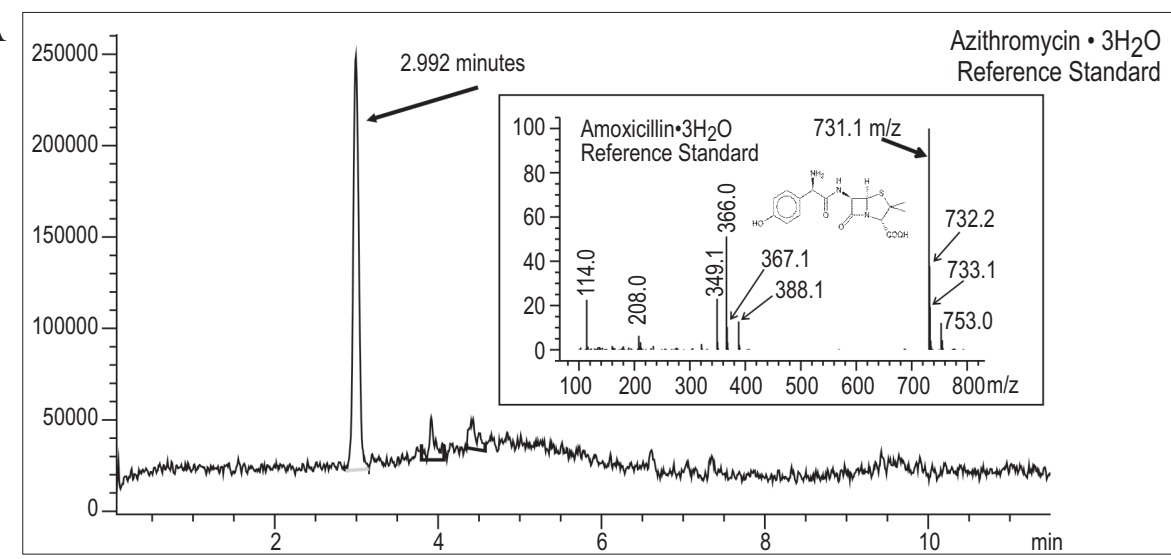

B

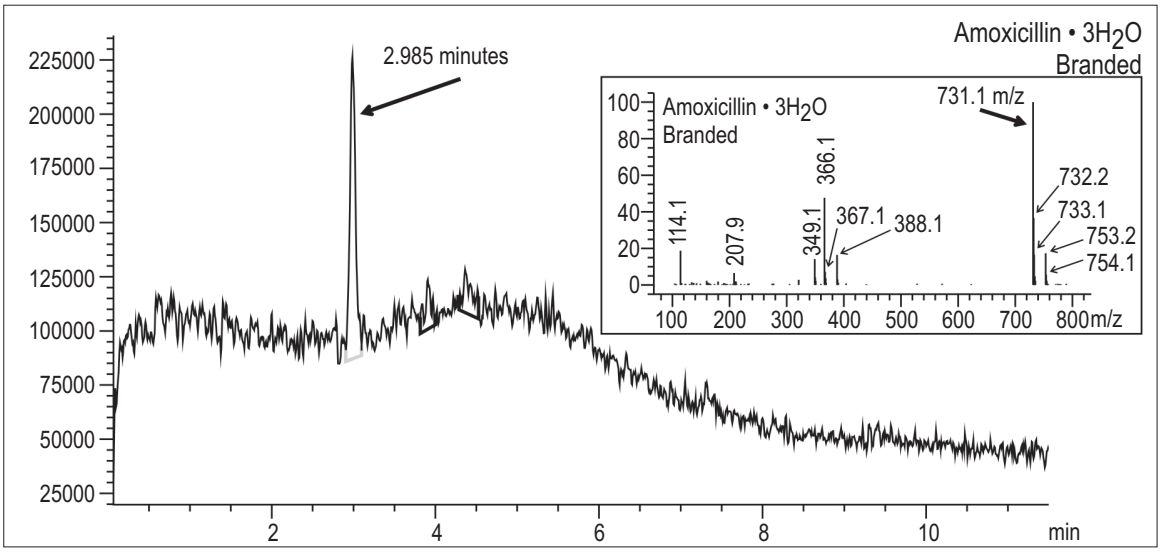




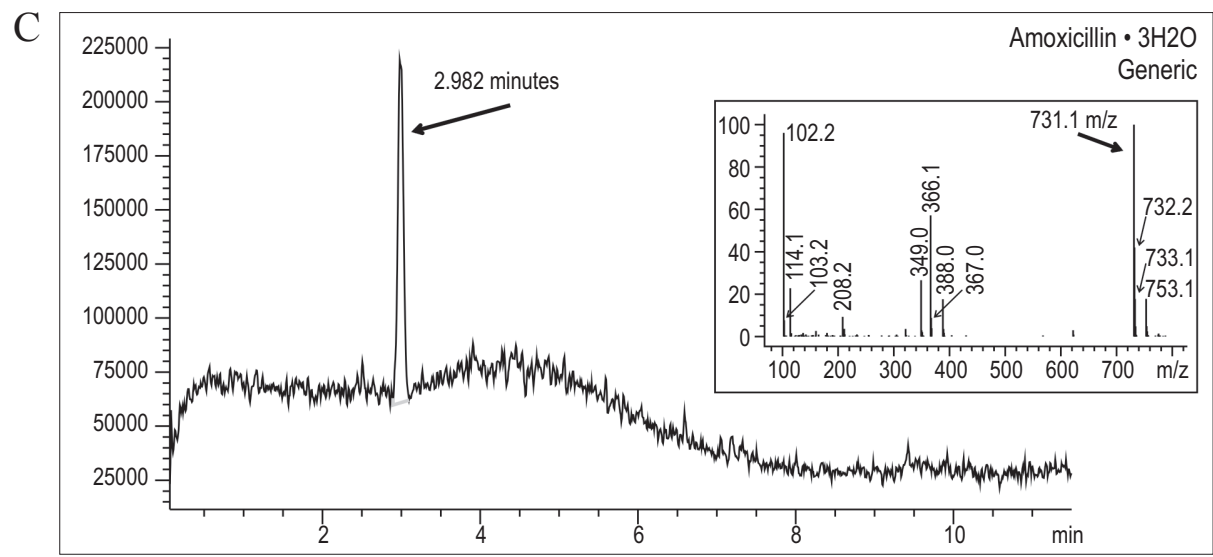

Figure 6 shows the chromatogram (LC-MS) and mass spectra associated with the standard and generic fluconazole.

Figure 6. Chromatogram obtained by LC-MS (SCAN mode) and mass spectra for standard fluconazole (A) and generic fluconazole, which the main peak corresponds to fluconazole (4.840 min) (B).
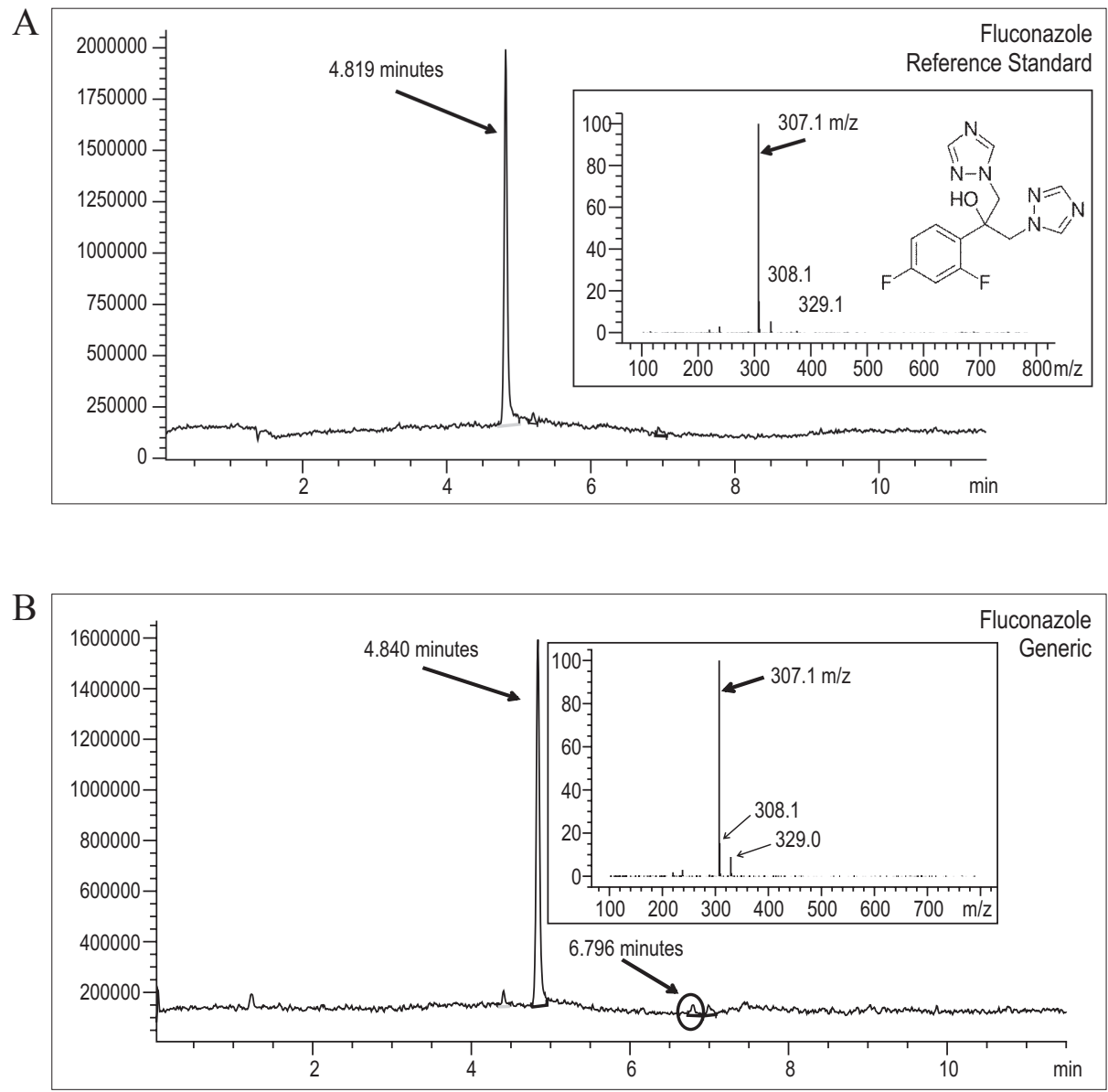
The peak at $4.840 \mathrm{~min}$ corresponds to fluconazole, as evidenced by the characteristic mass-to-charge ratio associated with fluconazole seen in the mass spectrum of this peak (Figure 6), in addition to the comparison with the pure standard of this substance. Additionally, there is a small peak at $6.796 \mathrm{~min}$, which did not have a matching peak in the pure standard. Further analysis of the mass spectrum of this peak (Figure 7) shows a fragment with a molecular weight of $227 \mathrm{~g}$ mol-1. A search of known impurities of fluconazole did not reveal any molecules with the same molecular weight, but it should be noted that this does not mean that any of the known impurities are not present.

Figure 7. Mass spectrum of $6.796 \mathrm{~min}$ peak for generic fluconazole.

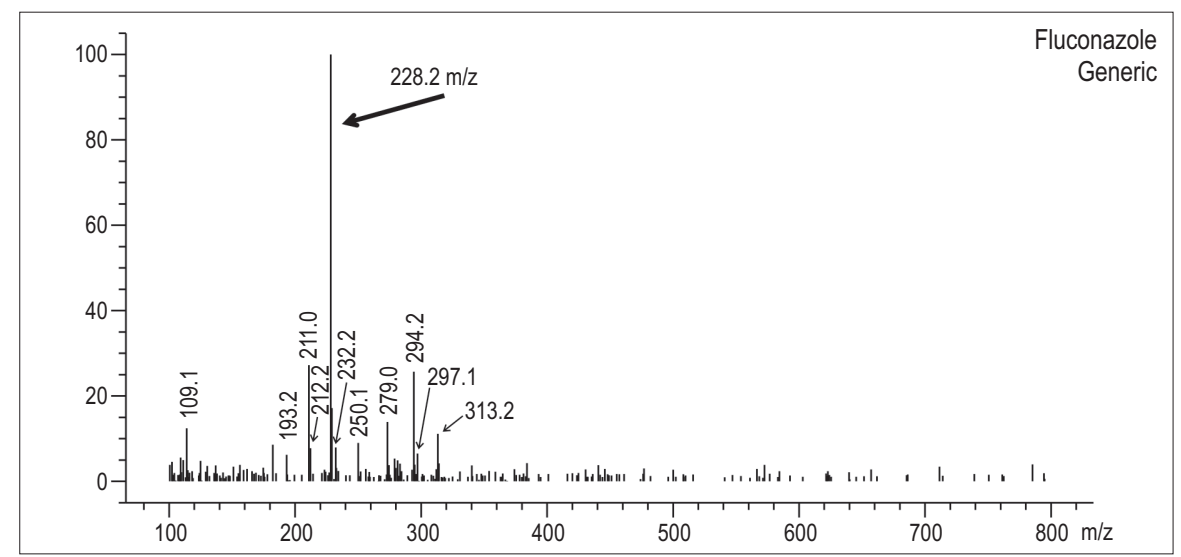

Figure 8 shows the mass spectrum for the pure itraconazole standard, which corresponds to the peak at $8.654 \mathrm{~min}$ from LC-MS chromatogram. The molecular weight of itraconazole is 705.65 $\mathrm{g}$ mol-1. There are also several peaks that differ only by one mass unit: 706.2, 707.2, 708.2, and $710.2 \mathrm{~m} / \mathrm{z}$, which indicate that itraconazole can ionize in several different ways, only differing by the mass of a few hydrogen atoms. It is essential to know when identifying possible impurities - the molecular weight indicated by a mass spectrum is not guaranteed to be the actual molecular weight of that particular chemical entity.

Figure 8. Chromatogram obtained by LC-MS (SCAN mode) and mass spectra for standard itraconazole.

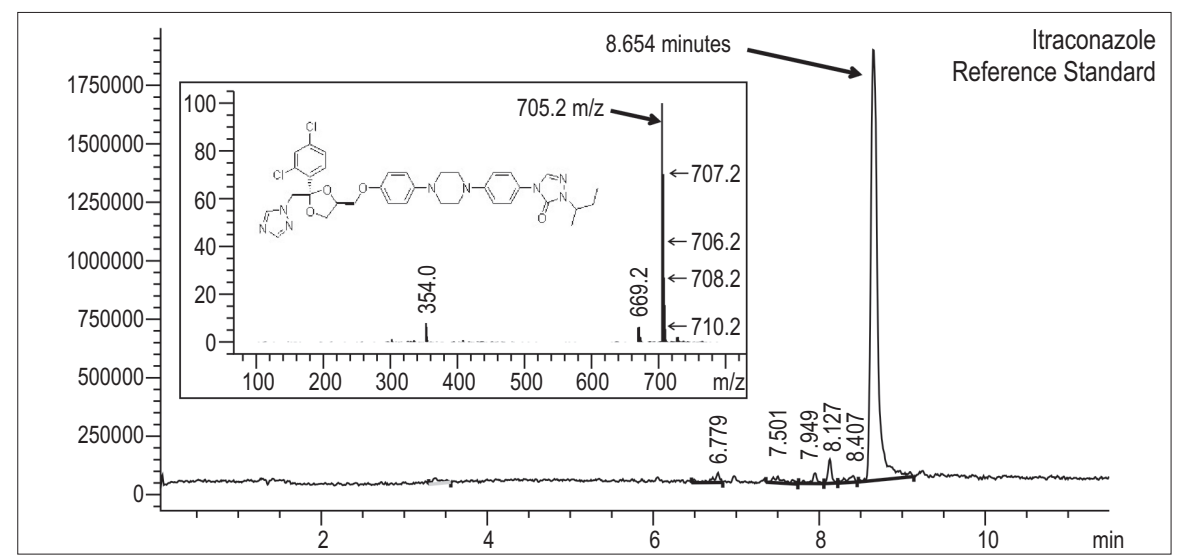


For the generic itraconazole, the peak at 8.663 min corresponds to itraconazole, as evidenced by the characteristic mass-to-charge ratios and pattern associated with itraconazole seen in the mass spectrum of this peak (Figure 9). There are several other small peaks (with retention times shown) that indicate chemical entities that have conjugated moieties. In particular, there is a small peak at $7.853 \mathrm{~min}$ on the LC-
MS scan, which does not have a matching peak in the standard. Analysis of the mass spectrum of this peak (Figure 9C) reveals the presence of a chemical entity with a primary fragment with a mass-to-charge ratio of $408.3 \mathrm{~m} / \mathrm{z}$. As mentioned earlier, it is unclear whether this corresponds to a molecular weight of $407 \mathrm{~g}$ mol-1 or $408 \mathrm{~g}$ mol-1 based on how itraconazole ionizes with ESI.

Figure 9. Chromatogram obtained by LC-MS (SCAN mode) for generic itraconazole (A), Mass spectrum of 8.663 min peak for generic fluconazole (B) and Mass spectrum of 7.853 min peak for generic fluconazole $(\mathrm{C})$.
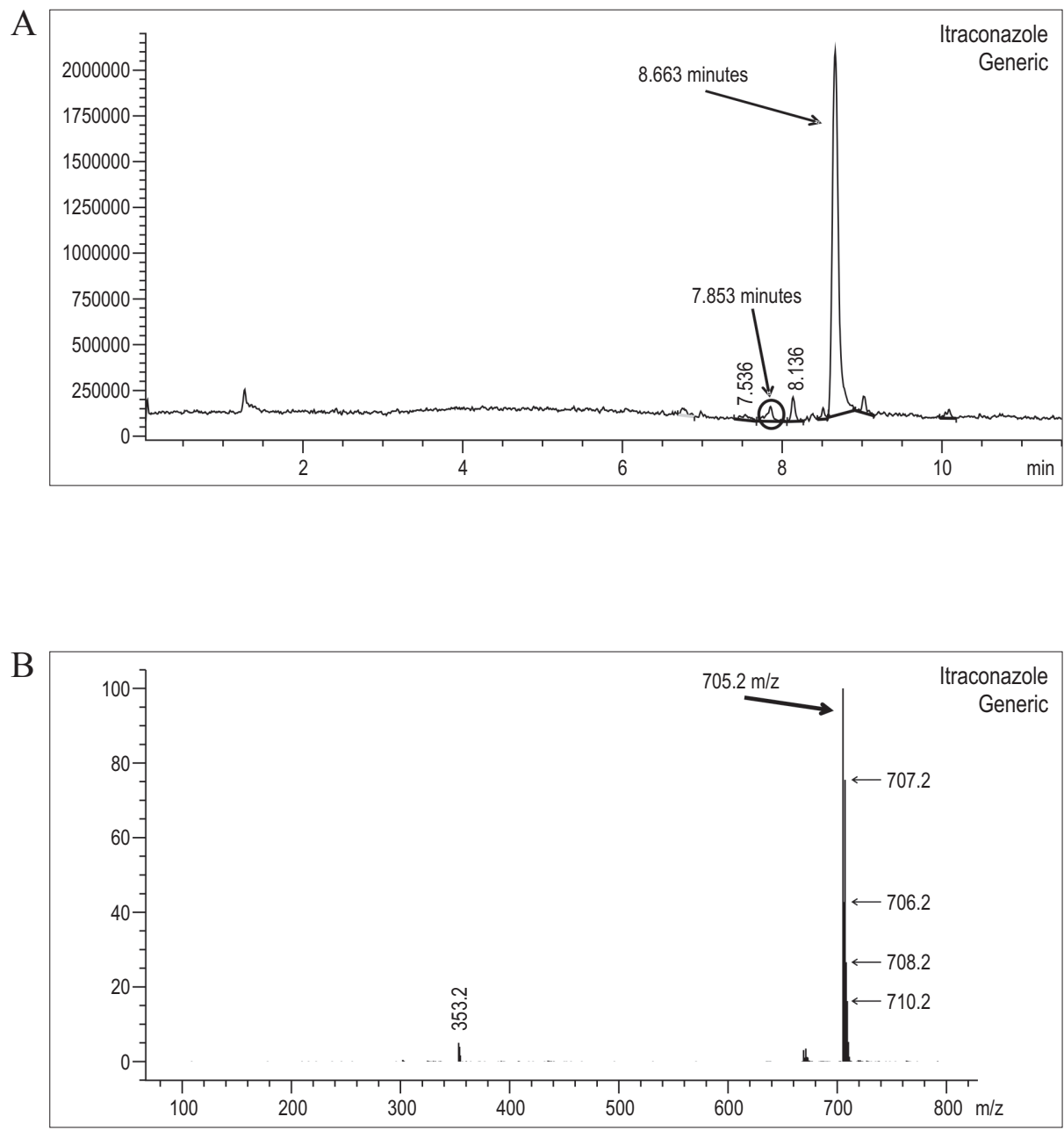


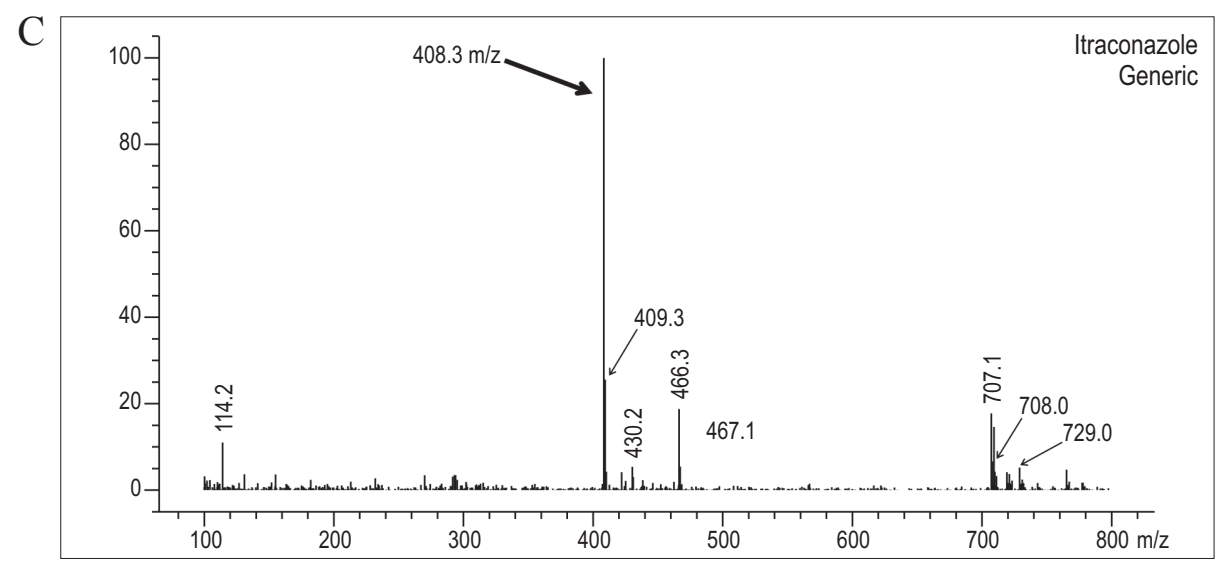

Wharton et al. in 2014 found different molecules with the mentioned molecular weights may be known impurities of itraconazole (21). Two molecules (Figure $10 \mathrm{~A}$ and B) are cistrans isomers: cis-[2-(2,4-Dichlorophenyl)-

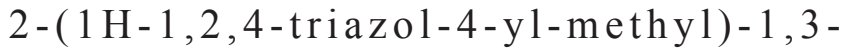
dioxolan-4-yl]methylmethanesulfonate, and trans-[2-(2,4-Dichlorophenyl)-2-(1H-1,2,4triazol-1-yl-methyl)-1,3-dioxolan-4-yl] methylmethanesulfonate, respectively. Both of these molecules have a molecular weight of $408.3 \mathrm{~g}$ mol-1. A third molecule (Figure 10C) is a known impurity according to the European Pharmacopoeia (itraconazole EP impurity A). This molecule is 2- (2-Butyl) -4-\{4-[4-(4-methyloxyphenyl)-piperazin-1-yl]-phenyl $\}-2,4$-dihydro[1,2,4]-triazol-3-one (CAS\#: 252964-68-4) and has a molecular weight of $407.5 \mathrm{~g}$ mol-1.

Figure 10. Structure of (A) cis-[2-(2,4-Dichlorophenyl)-2-(1H-1,2,4-triazol-4-yl-methyl)-1,3-dioxolan4-yl]methylmethanesulfonate (CAS\#: 67914-86-7); (B) trans-[2-(2,4-Dichlorophenyl)-2-(1H-1,2,4triazol-1-yl-methyl)-1,3-dioxolan-4-yl]methyl methanesulfonate (CAS\#: 854372-78-4) and (C) 2-(2Butyl)-4-\{4-[4-(4-methyloxy-phenyl)-piperazin-1-yl]-phenyl\}-2,4-dihydro-[1,2,4]-triazol-3-one(CAS\#: 252964-68-4), known impurities of itraconazole.
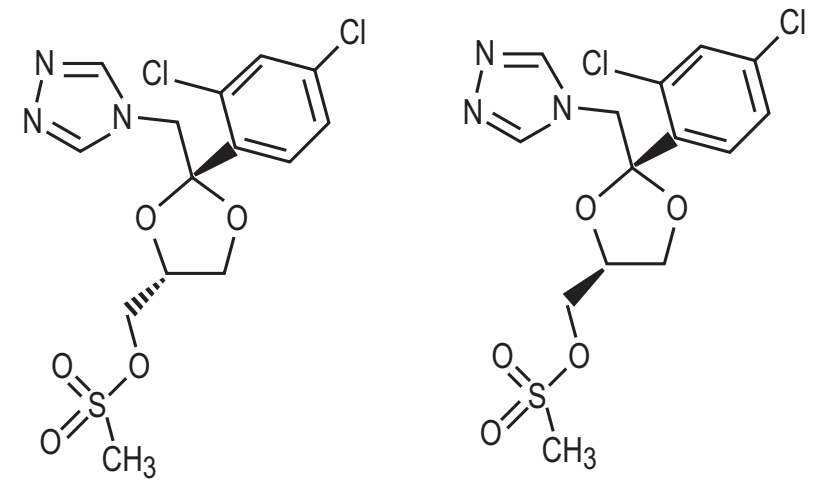<smiles>CCC(C)n1ncn(-c2ccc(N3CCN(c4ccc(OC)cc4)CC3)cc2)c1=O</smiles> 
These data indicate that the generic itraconazole appears to contain an impurity not found in a standard formulation of itraconazole, which and has a prominent mass fragment of $408.3 \mathrm{~m} / \mathrm{z}$.

Given the results discussed above, Table 1 shows a summary of the presence of organic impurities in generic and reference Brazilian medicines, as well as the evaluation of the API content present in each of the generic drugs analyzed.

\section{Conclusion}

We conclude that impurities which can interfere with the safety and efficacy of drugs are not routinely analyzed prior to registration for the treatment of human diseases. It is critical to explore the influence of such generic impurities with respect to their efficacy in addition to a bioequivalence test.

Table 1. Summary of the results of identification of impurities and quantification of the API of generic medicines compared to reference medicines.

\begin{tabular}{|c|c|c|c|c|}
\hline $\begin{array}{l}\text { Therapeutic } \\
\text { Class }\end{array}$ & $\begin{array}{l}\text { Compound } \\
\text { Description }\end{array}$ & $\begin{array}{l}\text { Sample } \\
\text { Impurity* }\end{array}$ & Possible & $\begin{array}{l}\% \text { API vs } \\
\text { Branded* }\end{array}$ \\
\hline \multirow[t]{3}{*}{ Antibiotic } & Amoxicillin trihydrate & Reference & ND & $\mathrm{n} / \mathrm{a}$ \\
\hline & & Generic 1 & ND & 97.7 \\
\hline & Azithromycin dihydrate & Generic 1 & $\begin{array}{l}\text { Azithromycin } \\
\text { EP impurity E, N }\end{array}$ & $\mathrm{n} / \mathrm{a}$ \\
\hline Antifungal & Fluconazole & Generic 1 & $\begin{array}{l}\text { Unknown (mass } \\
\sim 227 \text { g mol-1) }\end{array}$ & $\mathrm{n} / \mathrm{a}$ \\
\hline Itraconazole & Generic 1 & & $\begin{array}{l}\text { cis-trans isomers } \\
\text { of known impurity }\end{array}$ & $\mathrm{n} / \mathrm{a}$ \\
\hline
\end{tabular}

*ND = not detected; $\mathrm{n} / \mathrm{a}=$ not applied.

\section{References}

1. Jain, M., Srivastava, V., Kumar, R., Dangi, V., Hiriyanna S.G., et al. Determination of five potential genotoxic impurities in dalfampridine using liquid chromatography. J Pharmaceut Biomed. 2017;133:27-31.

2. Krakowska, B., Custers, D., Deconinck, E., Daszykowski, M. Chemometrics and the identification of counterfeit medicines-A review. J Pharm Biomed Anal. 2016;127:112-22.

3. Newton, P,N., Green, M.D., Fernandez, F.M. Impact of poor-quality medicines in the 'developing' world. Trends Pharmacol Sci. 2010;31(3):99-101.

4. Fernandez FM, Hostetler D, Powell K, Kaur H, Green MD, Mildenhall DC, et al. Poor quality drugs: grand challenges in high throughput detection, countrywide sampling, and forensics in developing countries. Analyst. 2011;136(15):3073-82.

5. Hollein, L., Kaale, E., Mwalwisi, Y.H., Schulze, M.H., Holzgrabe, U. Routine quality control of medicines in developing countries: Analytical challenges, regulatory infrastructures and the prevalence of counterfeitmedicines in Tanzania. Trac-Trend Anal Chem. 2016;76:60-70.
6. Puig-Junoy, J. Impact of European pharmaceutical price regulation on generic price competition: a review. Pharmacoeconomics. 2010;28(8):649-63.

7. Buckley, G. J., Gostin, L.O. Countering the problem of falsified and substandard drugs. The National Academies Press. 2013:351.

8. Corrao, G., Soranna, D., Arfe, A., Casula, M., Tragni, E., Merlino, L., et al. Are generic and brand-name statins clinically equivalent? Evidence from a real data-base. Eur J Intern Med. 2014;25(8):745-50.

9. Rawlins, M.D. Generic prescribing: unfinished business. The Lancet. 2015;385:219.

10. Deconinck, E., Sacre, P.Y., Courselle, P., De Beer, J.O. Chromatography in the Detection and Characterization of Illegal Pharmaceutical Preparations. J Chromatogr Sci. 2013;51(8):791-806.

11. Erturk S, Aktas ES, Ersoy L, Ficicioglu S. An HPLC method for the determination of atorvastatin and its impurities in bulk drug and tablets. J Pharmaceut Biomed. 2003;33(5):1017-23.

12. Novakova, L., Satinsky, D., Solich, P. HPLC methods for the determination of simvastatin and atorvastatin. TracTrend Anal Chem. 2008;27(4):352-67. 
13. Rodrigues, L.R., de Oliveira, D.N., Ferreira, M.S., Catharino, R.R. In situ assessment of atorvastatin impurity using MALDI mass spectrometry imaging (MALDIMSI). Analytica Chimica Acta. 2014;818:32-8.

14. Taluri, M.V.N.K., Kalyankar, A.R.S. Synchronized separation of atorvastatin — an antihyperlipidemic drug with antihypertensive, antidiabetic, antithrombotic drugs by RP-LC for determination in combined formulations. Journal of Pharmaceutical Analysis. 2012;2(4):285-92.

15. Wang, J., Luzum, J.A., Phelps, M.A., Kitzmiller, J.P. Liquid chromatography-tandem mass spectrometry assay for the simultaneous quantification of simvastatin, lovastatin, atorvastatin, and their major metabolites in human plasma. J Chromatogr B Analyt Technol Biomed Life Sci. 2015;983-984:18-25.

16. Culzoni, M.J., Dwivedi, P., Green, M.D., Newton P.N., Fernandez, F.M. Ambient mass spectrometry technologies for the detection of falsified drugs. Medchemcomm. 2014;5(1):9-19.
17. Katny, M., Frankowski, M. Impurities in Drug Products and Active Pharmaceutical Ingredients. Crit Rev Anal Chem. 2017;47(3):187-93.

18. Dispas A, Desfontaine V, Andri B, Lebrun P, Kotoni D, Clarke A, et al. Quantitative determination of salbutamol sulfate impurities using achiral supercritical fluid chromatography. J Pharmaceut Biomed. 2017;134:170-80.

19. Plumb, R.S., Jones, M.D., Rainville, P., Castro-Perez, J.M. The rapid detection and identification of the impurities of simvastatin using high resolution sub $2 \mathrm{mu}$ m particle LC coupled to hybrid quadrupole time of flight MS operating with alternating high-low collision energy. J Sep Sci. 2007;30(16):2666-75.

20. Chang, Y., Wang, L.X., Li, Y.P., Hu, C.Q.Factors Influencing the HPLC Determination for Related Substances of Azithromycin. J Chromatogr Sci. 2016;54(2):187-94.

21. Wharton, M., Geary, M., Sweetman, P., Curtin, L., O'Connor, N. Rapid liquid chromatographic determination of itraconazole and its production impurities. J Chromatogr Sci. 2014;52(3):187-94. 